\title{
Genetic Heterogeneity Profiling by Single Cell RNA Sequencing
}

2 Zilu Zhou, Graduate Group in Genomics and Computational Biology, University of Pennsylvania,

3 Philadelphia, PA, USA. zhouzilu@pennmedicine.upenn.edu

4 Bihui Xu, Department of Radiation Oncology, Parker Institute for Cancer Immunotherapy, Abramson

5 Family Cancer Research Institute, Perelman School of Medicine, University of Pennsylvania,

6 Philadelphia, PA, USA.

7 Andy Minn, Department of Radiation Oncology, Parker Institute for Cancer Immunotherapy, Abramson

8 Family Cancer Research Institute, Graduate Group in Cell and Molecular Biology, Perelman School of

9 Medicine, University of Pennsylvania, Philadelphia, PA, USA.

10 Nancy R Zhang*, Department of Statistics, The Wharton School, University of Pennsylvania, Philadelphia,

11 PA, USA.nzh@wharton.upenn.edu

$12 *$ Corresponding author 


\section{Abstract}

15 Detection of genetically distinct subclones and profiling the transcriptomic differences between them is

16 important for studying the evolutionary dynamics of tumors, as well as for accurate prognosis and

17 effective treatment of cancer in the clinic. For the profiling of intra-tumor transcriptional heterogeneity,

18 single cell RNA-sequencing (scRNA-seq) is now ubiquitously adopted in ongoing and planned cancer

19 studies. Detection of somatic DNA mutations and inference of clonal membership from scRNA-seq,

20 however, is currently unreliable. We propose DENDRO, an analysis method for scRNA-seq data that

21 detects genetically distinct subclones, assigns each single cell to a subclone, and reconstructs the

22 phylogenetic tree describing the tumor's evolutionary history. DENDRO utilizes information from single

23 nucleotide mutations in transcribed regions and accounts for technical noise and expression

24 stochasticity at the single cell level. The accuracy of DENDRO was benchmarked on spike-in datasets and

25 on scRNA-seq data with known subpopulation structure. We applied DENDRO to delineate subclonal

26 expansion in a mouse melanoma model in response to immunotherapy, highlighting the role of

27 neoantigens in treatment response. We also applied DENDRO to primary and lymph-node metastasis

28 samples in breast cancer, where the new approach allowed us to better understand the relationship

29 between genetic and transcriptomic intratumor variation.

\section{Keywords}

32 single-cell RNA sequencing, intratumor heterogeneity, cancer genomics, phylogeny inference, multi-

33 omics analysis 


\section{Background}

36 DNA alterations, especially single nucleotide alteration (SNA) and epigenetic modulation both contribute

37 to intratumor heterogeneity [1], which mediates tumor initiation, progression, metastasis and relapse

$38[2,3]$. Intratumor genetic and transcriptomic variation underlie patients' response to treatment, as

39 natural selection can lead to the emergence of subclones that are drug resistant [4]. Thus, identifying

40 subclonal DNA alterations and assessing their impact on intratumor transcriptional dynamics can

41 elucidate the mechanisms of tumor evolution and, further, uncover potential targets for therapy. To

42 characterize intratumor genetic heterogeneity, most prior studies have used bulk tumor DNA

43 sequencing [5-12], but these approaches have limited resolution and power [13].

44 Breakthroughs in single-cell genomics promise to reshape cancer research by allowing comprehensive

45 cell type classification and rare subclone identification. For example, in breast cancer, single-cell DNA

46 sequencing (scDNA-seq) was used to distinguish normal cells from malignant cells, the latter of which

47 were further classified into subclones [14-16]. For the profiling of intra-tumor transcriptional

48 heterogeneity, single cell RNA-sequencing (scRNA-seq), such as Smart-seq2 [17], Drop-seq [18], and 10X

49 Genomics Chromium ${ }^{\text {TM }}$, is now ubiquitously adopted in ongoing and planned cancer studies. ScRNA-seq

50 studies have already led to novel insights into cancer progression and metastasis, as well as into tumor

51 prognosis and treatment response, especially response variability in immune checkpoint blockade (ICB)

52 [19-26]. Characterization of intratumor genetic heterogeneity and identification of subclones using

53 scRNA-seq is challenging, as SNAs derived from scRNA-seq reads are extremely noisy and most studies

54 have relied on the detection of chromosome-level copy number aberrations through smoothed gene

55 expression profiles. Yet, as intratumor transcriptomic variation is partially driven by intratumor genetic

56 variation, the classification of cells into subclones and the characterization of each subclone's genetic

57 alterations should ideally be an integral step in any scRNA-seq analysis. 
58 The appeal of subclone identification in scRNA-seq data is compounded by the shortage of technology

59 for sequencing the DNA and RNA molecules in the same cell with acceptable accuracy, throughput, and

60 cost. Although one can apply both scDNA-seq and scRNA-seq to a given cell population, the mutation

61 analysis and RNA quantification cannot be conducted in the same set of cells. Although there are now

62 technologies for deep targeted sequencing of select transcripts matched with same-cell whole

63 transcriptome sequencing $[27,28]$, these methods are still, in effect, profiling DNA-level variation by

64 sequencing expressed transcripts, and are thus subject to the technical issues, especially dropout due to

65 transcriptional stochasticity, that we modeled in DENDRO.

66 Subclone detection using scRNA-seq is difficult mainly because only a small portion of the SNAs of each

67 cell is expected to be seen in the read output of scRNA-seq. This is because to be sequenced, an SNA

68 needs to fall in a transcribed region of the genome, at a location within the transcript that will

69 eventually be read by the chosen sequencing protocol. Even for SNAs that satisfy these requirements,

70 the mutated allele are often missing in the read output due to dropout, especially in the heterozygous

71 case. This is due, in part, to the bursty nature of gene transcription in single cells [29-31], where in any

72 given cell, a substantial fraction of the genes are only expressed from one of the alleles. Thus, an SNA

73 residing in a gene that is expressed at the bulk tissue level may not be observed in a particular cell,

74 simply because the mutated allele, by chance, is not expressed in the given cell. We refer to alleles that

75 are not captured due to expression stochasticity as biological dropouts. Even for a mutated allele that is

76 expressed, it has to be successfully converted to cDNA and then sequenced to be represented in the

77 final read output; we refer to alleles lost due to technical reasons as technical dropouts. In addition to

78 dropout events, post-transcriptional modification, such as RNA editing, and sequencing errors impede

79 both the sensitivity and the specificity of SNA discovery. As a result, methods developed for single cell

80 SNA detection using scDNA-seq, such as Monovar [32], as well as methods designed for SNA detection in

81 bulk DNA or RNA sequencing data do not yield accurate results in the scRNA-seq setting [33-38]. 
82 Here we present a new statistical and computational framework - NA based Evolution Nary tree

83 preDiction by scRNA-seq technology (DENDRO) - that reconstructs the phylogenetic tree for cells

84 sequenced by scRNA-seq based on genetic divergence calculated from DNA-level mutations. DENDRO

85 assigns each cell to a leaf in the tree representing a subclone, and, for each subclone, infers its mutation

86 profile. DENDRO can detect genetically divergent subclones by addressing challenges unique to scRNA-

87 seq, including transcriptional variation and technical noise. A DENDRO clustering of scRNA-seq data

88 allows joint genetic and transcriptomic analysis on the same set of cells.

89 We evaluate DENDRO against existing approaches, through spike-in data sets and a metastasized renal

90 cell carcinoma dataset with known subpopulation labels, and show that DENDRO improved the accuracy

91 of subclone detection. We then demonstrate the DENDRO to biological discovery through two

92 applications. The first application profiles the treatment response in a melanoma model to immune

93 checkpoint blockade therapy. DENDRO identified a subclone that contracted consistently in response to

94 ICB therapy, and revealed that the contraction was driven by the high mutation burden and increased

95 availability of predicted neoantigens. Transcriptional divergence between the subclones in this model

96 was very weak, and thus the neoantigen-driven sub-clonal dynamics would not have been detected

97 without extracting DNA-level information. In the second application to a breast tumor dataset, DENDRO

98 detected subclones and allowed for the joint characterization of transcriptomic and genetic divergence

99 between cells in lymph-node metastasis and cells in primary resections.

100 The DENDRO package, implemented in R, is available at https://github.com/zhouzilu/DENDRO, where

101 we also provide a power calculation toolkit, DENDROplan, to aid in the design of scRNA-seq experiments

102 for subclonal mutation analysis using DENDRO.

103 Results 


\section{Method overview}

106 Figure $1 \mathrm{~A}$ shows an overview of DENDRO's analysis pipeline. Per cell counts of total read coverage $(N$

107 matrix) and mutation allele read coverage ( $X$ matrix) at SNA locations are extracted after read alignment 108 and SNA detection (details in Methods, Figure S1). Based on these matrices, DENDRO then computes a

109 cell-to-cell genetic divergence matrix, where entry $\left(c, c^{\prime}\right)$ of the matrix is a measure of the genetic

110 divergence between cells $c$ and $c^{\prime}$. Details of this genetic divergence evaluation will be given in the next

111 section. DENDRO then clusters the cells into genetically distinct subclones based on this pairwise

112 divergence matrix, and selects the number of subclones based on inspection of the intra-cluster

113 divergence curve. Reads from the same subclone are then pooled together, and the SNA profile for each

114 subclone is re-estimated based on the pooled reads, which improves upon the previous SNA profiles

115 computed at the single cell level. Finally, DENDRO generates a parsimony tree using the subclone-level

116 mutation profiles to more accurately reflect the evolutionary relationship between the subclones.

\section{Genetic divergence evaluation}

118 Due to the high rates of biological and technical dropout, SNA detection within each individual cell lacks

119 sensitivity. We also expect low specificity due to the high base error rate in scRNA-seq protocols. Thus,

120 simple distance measures such as the Hamming or Euclidean distances evaluated on the raw SNA

121 genotype matrix or the raw allele frequency matrix do not accurately reflect the genetic divergence

122 between cells.

123 To more accurately estimate the cell-to-cell genetic divergence, we have developed a statistical model

124 that accounts for technical dropout, sequencing error and expression stochasticity. Consider two cells, $c$

125 and $c^{\prime}$, and let $I_{c}$ and $I_{C^{\prime}}$ index the clonal group to which the cells belong. That is, $I_{c}=I_{C^{\prime}}$, if cells $c$ and $c^{\prime}$ 
come from the same subclone and thus share the same SNA profile. Let $X_{c}=\left(X_{c 1}, \ldots, X_{c m}\right)$ be the

127 mutation allele read counts for this cell at the $m$ SNA sites profiled, and $N_{c}=\left(N_{c 1}, \ldots, N_{c m}\right)$ be the total read counts at these sites. We define the genetic divergence between the two cells as

$$
d_{c c^{\prime}}=-\log P\left(X_{c}, X_{c^{\prime}} \mid N_{c}, N_{c^{\prime}}, I_{c}=I_{c^{\prime}}\right)=\sum_{g=1}^{m} d_{c c^{\prime}}^{g}
$$

$$
\text { where } d_{c c^{\prime}}^{g}=-\log P\left(X_{c g}, X_{c^{\prime} g} \mid N_{c g}, N_{c^{\prime} g}, I_{c}=I_{c^{\prime}}\right)
$$

131 In other words, $d_{c c^{\prime}}$ is the negative log likelihood of the mutation allele counts of cells $c$ and $c^{\prime}$, given

132 the total read counts and the event that the two cells belong to the same subclone. If $c$ and $c^{\prime}$ have

133 mutations in mismatched positions, this likelihood for $X_{c}, X_{c^{\prime}}$ conditioned on $I_{c}=I_{c^{\prime}}$ would be small,

134 giving a large value for $d_{c c^{\prime}}$. By the assumption of independence between sites, $d_{c c^{\prime}}$ is the sum of $d_{c c^{\prime}}^{g}$,

135 where $d_{c c^{\prime}}^{g}$ is the contribution of mutation site $g$ to the divergence measure. In characterizing the

136 conditional distribution for $X_{c g}$ and $X_{c^{\prime} g}$, we use a Beta-Binomial distribution to model expression

137 stochasticity and a Binomial model to capture sequencing errors and rare RNA-editing events. Referring

138 to Figure $1 \mathrm{~b}$, mutations residing in bursty genes, such as gene $g$, would tend to have U-shaped allele

139 frequency distributions and are more likely to be "dropped" due to low or zero expression. In contrary,

140 mutations residing in constitutive (non-bursty) genes, such as gene $g$ ' in Figure $1 \mathrm{~b}$, would have bell-

141 shaped allele frequency distributions and can be genotyped more reliably. Thus, even if the read counts

142 for the mutation loci residing in genes $g$ and $g$ 'are identical across two cells ( $c_{1}$ and $c_{2}$ in Figure $1 \mathrm{~b}$ ),

143 the locus in $g^{\prime}$ would contribute a higher value, compared to the locus in $g$, to the divergence between

144 cells $c_{1}$ and $c_{2}$. Please see Methods for details.

\section{Accuracy assessment}


147 First, we designed a spike-in procedure to assess the accuracy of DENDRO versus existing approaches

148 and to make realistic power projections for subclone detection (Figure 2a). Since DENDRO is currently

149 the only method for SNA-based subclone detection using scRNA-seq data alone, we benchmarked

150 against more straightforward approaches such as hierarchical clustering based on mutation allele

151 frequencies. The spike-in procedure starts with an assumed evolutionary tree, where the leaves are

152 subclones and mutations can be placed on the branches. In the absence of prior information, a simple

153 tree structure is used, such as the one shown in Figure 2A. Parameters of simulation are (1) total

154 number of mutations, (2) total number of cells, (3) the proportion of cells in each clade, (4) the

155 proportion of mutations along each branch, and (5) mean read coverage across loci. Some of these

156 parameters can be determined using bulk DNA-seq and/or bulk RNA-seq data if available (Methods).

157 Parameters (1-4) determine the mutation profile matrix $Z$. To get the matrix of alternative and total

158 read counts for each mutation loci in each cell ( $X_{c g}$ and $N_{c g}$, respectively), we overlay the mutation

159 matrix onto the scRNA-seq data. This allows the spike-in framework to retain the expression

160 stochasticity and sequencing error of real scRNA-seq data. DENDRO is then applied to the read count

161 matrices to obtain the subclone clusters, which is then compared with the known labels. Accuracy is

162 evaluated by three metrics: adjusted Rand index, capture rate and purity (Supplementary Materials).

163 Such spike-in procedure can also facilitate experiment design, as it predicts the expected clustering

164 accuracy by DENDRO given sequencing parameters and available bulk data for the tumor (See

165 DENDROplan in Methods).

166 Using the above framework, we conducted a systematic evaluation of DENDRO's subclone detection

167 accuracy on an example scRNA-seq dataset with allelic information [39]. The results, compiled in Figure

$1682 \mathrm{~b}$, Supplementary Materials and Figure S3, show that DENDRO has better performance than simply 
clustering on mutation allele frequencies and quantify how accuracy depends on the mutation burden,

170 mutation read depth, mutation distribution, subclone cell proportion, and cell populations. Even when

171 there are only 100 mutations with relatively low average coverage (read depth equals to 1), DENDRO

172 can still extract meaningful clustering results (average $A R I \approx 0.8$ ). More importantly, variation in total

173 expression of genes does not influence DENDRO's divergence measure. DENDRO shows consistent

174 results in spike-in analysis between populations of single cell type and multiple cell types (Figure S3).

175 This is due to DENDRO's reliance only on the distribution of the mutation allele frequency conditioned

176 on the total read coverage, as illustrated by the simulation study (Supplementary Material and Figure

177 S2). The divergence evaluation reflects solely genetic distance not transcriptomic difference, allowing for

178 easy interpretation. A more extensive spike-in analysis can be found in the Supplementary Materials.

180 We also benchmarked DENDRO against existing methods on the renal cell carcinoma dataset from Kim

181 et al [21] (Figure 2C). This dataset contained 116 cells sequenced using the Smart-seq technology [17],

182 obtained from three tumors derived from one patient: a patient-derived xenograft (PDX) from the

183 primary renal cell carcinoma (PDX_pRCC), a biopsy of the metastasis to the lung 1 year after treatment

184 of primary site (Pt_mRCC), and a PDX of the lung metastasis renal cell carcinoma (PDX_mRCC) (Figure

185 S4A). The cells should share common early driver mutations due to their shared origin from the same

186 patient, but the metastasis and the cultivation of each tumor in separate medium (human or mouse)

187 should have allowed for the accumulation of new mutations. Thus, we expect the three tumors to be

188 clonally distinct. This knowledge allows us to use this dataset to benchmark accuracy and to illustrate

189 how DENDRO enables joint analysis of the genetic and transcriptomic heterogeneity at single cell

190 resolution. 
191 We compared 4 different clustering methods: (1) DENDRO, (2) hierarchical clustering based on the

192 primary genotype matrix $Z$ generated by GATK ( $Z_{c g}=1$ when a mutation $g$ is detected for cell $c, Z_{c g}=$

1930 otherwise), (3) hierarchical clustering based on the $X / N$ matrix that preserve the variant allele

194 information and (4) hierarchical clustering based on gene expression ( $\log$ TPM). DENDRO gives the

195 cleanest separation between the three populations with adjusted Rand Index of 0.932 (1.0 indicates

196 perfect clustering, Figure 2C panel 1), as compared to 0.754 for Z matrix (Figure 2C panel 2), 0.519 for

$197 \mathrm{X} / \mathrm{N}$ (Figure 2C panel 3) and 0.489 for expression (Figure 2C panel 4). Inspection of the tree shows that,

198 as expected, divergence between primary tumor and metastasis exceeds divergence between patient

199 sample and PDX sample, as PDX_mRCC clusters with Pt_mRCC rather than PDX_pRCC. All of the other

200 three methods successfully separated the primary sample from the metastatic samples, but could not

201 differentiate between the two metastasis samples.

202 For DENDRO, the intra-cluster divergence curve flattened at 3, and thus we stopped splitting at 3

203 clusters (Figure S4E and Methods). We annotated the clusters as PDX_mRCC, PDX_pRCC and Pt_mRCC

204 by their cell compositions (Table S3A). DENDRO found minimal sharing of subclones among the tumors

205 derived from three sources, and low genetic heterogeneity within each tumor. This is unsurprising since

206 relapsed metastasis consists of cells that have already undergone selection, and since the PDX tumors

207 are each seeded by a small subsample of cells from the original tumor, each tumor consists of unique

208 subclones not detected in other sites [40-42]. Additional joint analysis of transcriptome and DNA

209 mutations can be found in the Supplementary Materials.

DENDRO analysis of melanoma model in response to immune checkpoint blockade highlights

\section{1 the role of neoantigens}

212 Immune checkpoint blockade (ICB) of the inhibitory receptors CTLA4 and PD1 can result in durable 213 responses in multiple cancer types [43]. Features intrinsic to cancer cells that can impact ICB treatment 
214 outcome include their repertoire of neoantigens [44], tumor mutational burden (TMB) [45], and

215 expression of PDL1 [46]. DENDRO analysis of scRNA-seq data allows joint DNA-RNA analysis of single

216 cells, thus enabling the simultaneous quantification of tumor mutational burden, the prediction of

217 neoantigen repertoire, and the characterization of gene expression profile at subclonal resolution. Thus,

218 to demonstrate the power of DENDRO and to better understand the relationship between ICB response

219 and intratumor heterogeneity, we profiled the single cell transcriptomes across three conditions derived

220 from 2 melanoma cell lines (Figure 3A): B16 melanoma cell line, which has shown modest initial

221 response to ICB treatment but eventually grows out, and Res 499 melanoma cell line (R499), which was

222 derived from a relapsed B16 tumor after combined treatment of radiation and anti-CTLA4 and is fully

223 resistant to ICB [47]. B16 was evaluated with and without anti-PD1 treatment, as we wanted a tumor

224 model that captures a transient ICB response. A total of 600 tumor cells were sequenced with Smart-seq

225 technology from six mice across three conditions: two mice with B16 without treatment (B16), two mice

226 with B16 after anti-PD1 treatment (B16PD1) and two mice with R499 without treatment (R499) (Figure

$2273 \mathrm{~A}$ and Methods). The existence of multiple subclones in B16 and R499 was suggested by bulk WES

228 analysis $[47,48]$. Our goal here is to determine whether the subclones differ in anti-PD1 response, and if

229 so, what are the subclonal differences.

230 A DENDRO analysis of 4059 putative mutation sites across 460 cells retained after QC (see Methods and

231 Figure S9A, B, C) yields the clustering displayed in Figure 3B, with four subclones suggested by the intra-

232 cluster divergence curve (Figure S9D). All subclones are shared among the three conditions, which is not

233 unexpected given that all tumor cells were derived from the same parental cell line. However, the sub-

234 clonal proportions vary significantly between conditions (Figure 3B). The subclonal proportions of

235 B16PD1 are approximately intermediate between that of B16 and R499. This is expected as R499 had

236 gone through immune editing whereas B16PD1, at the time of harvest, was still undergoing immune

237 editing and was at the transient response state. Furthermore, the selective pressure of radiation plus 
anti-CTLA4 is likely more than that of anti-PD1 treatment, as the former but not the latter results in

239 complete responses in our B16 model [47]. The frequency of Clone 2 is lower in B16PD1 and R499,

240 indicating sensitivity to anti-PD1 treatment, while the frequencies of Clone 3 and Clone 4 increase after

241 treatment and are the highest in R499, indicating resistance to therapy (Figure 3C, S10A).

242 To explore why subclones vary in sensitivity to anti-PD1 treatment, we compared the mutation profile of

243 Clone 2 to the other subclones. We pooled cells in each of the four subclones and re-estimated their

244 mutation profiles, which were then used to construct a phylogenetic tree (Figure 3D). The phylogeny

245 suggests that Clone 3 and Clone 4 are genetically closer to each other than to Clone 2, and thus, their

246 similarity in treatment response may be in part due to similarity in their mutation profiles. The re-

247 estimated mutation profiles show that Clone 2 has the highest tumor mutation burden, which has been

248 associated with increased likelihood of ICB response $[49,50]$. We then predicted the quantity of high-

249 affinity $(\leq 100 \mathrm{~nm}$ ) neoantigens in each subclone given its mutation profile [48]. As shown in Figure 3E,

250 Clone 2 has twice as many high-affinity neoantigens as the other three subclones. The high level of

251 neoantigens can lead to better T cell recognition, resulting in increased efficacy of anti-PD1 treatment

$252[51]$.

253 Analysis of gene expression, on the other hand, did not yield detectable known signatures associated

254 with anti-PD1 treatment sensitivity. Projections based on the expression of highly variable genes, as

255 shown in PCA and t-SNE plots (Figure S8), did not yield meaningful clusters. Differential expression

256 analysis between each subclone and the other subclones found few genes with adjusted P-value $<0.05$,

257 indicating similar expression across sub-clones that is concordant with the lack of structure in the

258 expression PCA and tSNE plots. Expressions of PdI1 (aka. Cd274) showed no differences between

259 subclones (KS-test: P-value > 0.42, Figure S10B). In addition, there were no detectable chromosome-

260 level differences in smoothed gene expression, indicating that there are no large CNV events that 
261 distinguish the subclones (Figure S11). DENDRO, detecting exonic mutations from scRNA-seq data,

262 enabled the finding of subclones in this data, the prediction of neoantigen load of each subclone, and

263 the analysis of subclonal dynamics due to treatment. Our analysis suggests that the genetic

264 heterogeneity, rather than transcriptomic heterogeneity, contributes to treatment efficacy in this tumor

265 model.

267 Simultaneous analysis of genetic and transcriptomic variation in single cell breast cancer

268 We next applied DENDRO to the analysis of data from a study of primary and metastasized breast cancer

269 [20]. We focused on two tumors (BC03 and BC09) that had the most cells sequenced (Table S5 and

270 Figure S12). BC03 included cells from the primary tumor (here after BC03P) as well as cells from regional

271 metastatic lymph nodes (here after BC03LN), whereas BC09 included cells only from the primary

272 resection. 132 single cell transcriptomes were profiled by Smart-seq protocol [17]. We first assess

273 whether DENDRO separated BC03 and BC09, and then examine the transcriptomic and genetic

274 heterogeneity within each tumor.

275 GATK [52] detected a total of 2,364,823 mutation sites across the 132 cells, 353,647 passed QC and were

276 retained for downstream analysis (Figure S12A, B, C). Figure 4 shows the clustering determined by

277 DENDRO. DENDRO separates BC09 cells from BC03 cells with $100 \%$ accuracy (Figure 4A). The intra-

278 cluster divergence curve flattened at five subclones: three subclones for BC03 and two for BC09 (Figure

279 4A, Figure S12D and Table S3B). Within BC03, Clone Mix_1 and Clone Mix_2 contained a mixture of cells

280 from the primary tumor and lymph nodes, and Clone LN_1 contained mostly cells from the lymph nodes.

281 This suggests that tumor cells that have metastasized to the lymph nodes are genetically

282 heterogeneous, with some cells remaining genetically similar to the primary population and others 
acquiring new genetic mutations. In comparison, hierarchical clustering based on expression (using log

transcripts-per-million values) did not separate $\mathrm{BCO} 3$ from $\mathrm{BC} 09$, and gave a negative adjusted Rand index within $\mathrm{BCO3}$, indicating effectively random assignment of cells to the two spatial locations (Figure 4B).

287 We then pooled cells within each of the 5 clusters and re-estimated their mutation profiles with

288 DENDRO. We defined a variant as subclonal if it was not present in all of the subclones within a tumor.

Based on detection marginal likelihood, we picked the top 10,000 most confident variants to construct a phylogenetic tree (Figure 4C). As expected, the two BC09 clusters are far from the three BC03 clusters.

291 Within $\mathrm{BCO3}$, the length of the branches shows that the subclone containing mostly cells from lymph

292 nodes (labeled BC03LN_1) is genetically more similar to Clone Mix_2 compared to Clone Mix_1 (Figure

293 4C). In addition, window-smoothed expression plot with cells grouped by DENDRO clustering shows

294 broad chromosome-level shifts in expression patterns between subclones, most likely due to copy

295 number aberrations that are consistent with SNAs (Figure S13) [22].

A comparison of the transcriptomes of the subclones revealed substantial differences in the expression

297 of PAM50 genes, which are prognostic markers for breast cancer (Figure 4D) [53]. DENDRO detected one

298 rare subclone, BC09_2, with only six cells ( $<5 \%$ of the total number of cells) which had a strong basal-like

299 signature. Interestingly, in BC03, Clone LN_1 has the TNBC/basal-like subtype with an invasive gene

300 signature, while Clone Mix_2 has the ESR1+ subtype. Thus, the genetic divergence of Clone LN_1 from

301 Clone Mix_2 is accompanied by its acquisition of an invasive metastatic expression signature. In a direct

302 comparison between cells from the primary site and cells from the lymph node without distinguishing

303 subclones, these expression differences would be much weaker since the subclones do not cleanly

304 separate by site. Compared with the original analysis that assigned each tumor to one specific breast

305 cancer subtype, this analysis identifies subclones with different expression phenotypes, potentially 
allowing for better therapy design that targets all subclone phenotypes to reduce the risk of tumor

307 relapse.

308 Existing scRNA-seq studies of cancer tissue cluster cells based on total gene expression or copy number

309 profiles derived from smoothed total expression, making it difficult to separate the effects of sub-clonal

310 copy number aberrations from transcriptomic variation [19, 22, 24]. Differential expression analysis

311 based on clusters derived from total expression is prone to self-fulfilling prophecy, as there would

312 indeed be differentially expressed genes because this is the clustering criteria. Because DENDRO's

313 subclone identification is based solely on genetic divergence, and not on expression profile, the

314 downstream differential gene expression analysis can be precisely attributed to transcriptional

315 divergence between subclones.

316 Hence, we conducted a transcriptome-wide search for pathways that have differential expression

317 between subclones (Methods and Table S6), and assessed their overlap with pathways that are

318 differentially mutated between subclones. Focusing on tumor BC03, pathways for G2M checkpoint and

319 KRAS signaling are up-regulated in lymph node metastasis Clone BCO3LN_1, while pathways for estrogen

320 response and apoptosis are down-regulated, indicating a more invasive phenotype (Table S6E). In

321 addition, GAPDH is up-regulated in the metastatic subclone (BCO3LN_1) and down-regulated in the two

322 mix-cell subclones, consistent with previous findings [54, 55] (Figure S14D). Differentially expressed

323 genes between other subclone pairs in $\mathrm{BCO} 3$ are also enriched in estrogen response, apoptosis, and DNA

324 repair (Table S6C, D). In parallel, subclone-specific mutated genes are highly enriched in cancer-related

325 pathways including MYC target, G2M checkpoints and mitotic spindle, and immune related pathways

326 such as, interferon response, TNF-a signaling and inflammatory response (Table S6). Interestingly, few of

327 the differentially mutated genes are associated with estrogen and androgen responses, suggesting that

328 the differential expression of hormone related genes is not mediated directly by genetic mutations in 
these pathways. This is consistent with the recent studies that epigenetic alteration, such as histone

330 acetylation and methylation, regulate hormones receptor signaling in breast cancer [56-59]. DNA-RNA

331 joint analysis between other subclones are included in the Table S6 and Figure S14. Overall, this example

332 illustrates how DENDRO enables the joint assessment of genetic and transcriptomic contributions to

333 clonal diversity at single-cell resolution.

\section{Discussion}

335 We have described DENDRO, a statistical framework to reconstruct intratumor DNA-level heterogeneity 336 using scRNA-seq data. DENDRO starts with mutations detected directly from the scRNA-seq reads, which

337 are very noisy due to a combination of factors: (1) errors are introduced in reverse-transcription,

338 sequencing and mapping, (2) low sequencing depth and low molecule conversion efficiency leading to

339 technical dropouts, and (3) expression burstiness at the single cell level leading to biological dropouts.

340 DENDRO overcomes these obstacles through the statistical modeling of each component. Given noisy

341 mutation profiles and allele-specific read counts, DENDRO computes a distance between each pair of

342 cells that quantifies their genetic divergence after accounting for transcriptional bursting, dropout and

343 sequencing error. Then, DENDRO clusters the cells based on this distance as subclone and re-estimates a

344 more robust subclone-specific mutation profile by pooling reads across cells within the same cluster.

345 These re-estimated mutations profiles are then passed to downstream mutation analysis and

346 phylogenetic tree reconstruction.

347 Importantly, the genetic divergence used by DENDRO for cell clustering is based solely on allelic

348 expression ratios and do not reflect the difference in total expression between cells at mutation sites.

349 Thus, DENDRO differs from, and complements, existing tools that cluster cells based on total expression.

350 In fact, as shown by spike-in analysis, DENDRO clusters the cells based on true underlining mutation

351 profiles, and is robust to changes in total gene expression. As expected, the numbers of cells, the depth 
352 of sequencing, the actual number of subclonal mutations and the phylogenetic tree structure all

353 influence the power of DENDRO. To aid researchers in experiment design, we developed DENDROplan,

354 which predicts DENDRO's clustering accuracy given basic experimental parameters and the expected

355 informative mutation count, which can be obtained from bulk DNA sequencing.

356 Ideally, joint sequencing of the DNA and RNA on the same cells would allow us to relate genomic profiles

357 to transcriptomic variations. Currently, there is yet no scalable technology for doing this. Separately

358 performing scDNA-seq and scRNA-seq on different batches of cells within the same tumor would meet

359 the nontrivial challenge of matching the subclones between the two data sets. DENDRO takes advantage

360 of the central dogma and utilizes computational methods to extract genetic divergence information

361 from noisy mutation calls in coding regions. Through two case studies, we illustrate the insights gained

362 from the subclonal mutation and expression joint analysis that DENDRO enables.

363 We have demonstrated that proper computational modeling can excavate the DNA-level heterogeneity

364 in scRNA-seq data. Yet, there is always limitation in working with RNA. While rare RNA editing events are

365 absorbed by the parameter $\epsilon$, DENDRO cannot distinguish subcluster-specific constituent RNA editing

366 events from subcluster-specific DNA mutations. In the extreme and unlikely scenario where RNA editing

367 events are common and pervasive, DENDRO's cluster would reflect RNA editing. In such cases, we

368 recommend using matched bulk DNA-seq of the same tumor to filter the loci detected in the first step of

369 DENDRO, keeping only those that are supported by at least on read in the bulk DNA-seq data.

371 Conclusions

372 We have developed DENDRO, a statistical method for tumor phylogeny inference and clonal

373 classification using scRNA-seq data. DENDRO accurately infers the phylogeny relating the cells and 
374 assigns each single cell from the scRNA-seq data set to subclone. DENDRO allows us to (1) cluster cells

375 based on genetic divergence while accounting for transcriptional bursting, technical dropout and

376 sequencing error, as benchmarked by in silico mixture and a spike-in analysis, (2) characterize the

377 transcribed mutations for each subclone, and (3) perform single-cell multi-omics analysis by examining

378 the relationship between transcriptomic variation and mutation profile with the same set of cells. We

379 evaluate the performance of DENDRO through a spike-in analysis and a data set with known subclonal

380 structure. We further illustrate DENDRO through two case studies. In the first case study of relationship

381 between intratumor heterogeneity and ICB treatment response, DENDRO estimates tumor mutation

382 burden and predicts repertoire of high-affinity neoantigens in each subclone from scRNA-seq. In the

383 second case study on a primary breast tumor dataset, DENDRO brought forth new insights on the

384 interplay between intratumor transcriptomic variation and subclonal divergence.

\section{Methods}

Figure S1 illustrates the SNA calling pipeline. Raw scRNA-seq data is aligned by STAR 2-pass method,

389 which accounts for splicing junctions and achieve higher mapping quality [60]. In the next step, raw

390 variants calling is made using the Haplotype Caller (GATK tool) on the BAM files after sorting, joining

391 read groups, removing duplicated reads, removing overhangs into intronic regions, realigning and

392 recalibration [61]. Conventionally, there are two methods from GATK tools for mutation detection:

393 haplotype caller and mutect2. Haplotype caller has a RNA-seq setting which handle splice junctions

394 correctly, but assumes VAF around 50\%, while mutect2 can detect mutations with low VAF but does

395 account for splice junction. The reason we select haplotype caller instead of mutect2 is that we extract 
allele read counts for all cells as long as one of the cells is listed as carrying the mutation. Thus, as long

397 as one cell has VAF reaching $50 \%$, this mutation would be detected. Calls with stand_call_conf greater

398 than 20 and population frequency greater than $5 \%$ but less than $95 \%$ were preserved for further

399 analysis. Admittedly, such lenient filtering typically introduces false positive sites. However, our priority

400 at this step is to minimize false negative rate, while the genetic divergence matrix in the following step

401 robustly estimates cell population substructure. Both the coverage of the alternative allele and the total

402 read coverage are extracted for each site for further analysis.

\section{Genetic Divergence and Beta-Binomial framework}

Consider two cells: $c$ and $c^{\prime}$. Let $I_{c}$ and $I_{c^{\prime}}$ denote the clonal group to which the cells belong, i.e. $I_{c}=I_{c^{\prime}}$

$407 \quad g$, by $d_{c c}^{g}$ :

$$
d_{c c^{\prime}}^{g}=\log \frac{1}{P\left(X_{c g}, X_{c^{\prime} g} \mid N_{c g}, N_{c^{\prime} g}, I_{c}=I_{c^{\prime}}\right)}
$$

$$
\left.=\log \frac{\mathrm{P}\left(X_{c g}, X_{c^{\prime} g} \mid N_{c g}, N_{c^{\prime} g}, I_{c}=I_{c^{\prime}}\right)+\mathrm{P}\left(X_{c g}, X_{c^{\prime} g} \mid N_{c g}, N_{c^{\prime} g}, I_{c} \neq I_{c^{\prime}}\right)}{P\left(X_{c g}, X_{c^{\prime} g} \mid N_{c g}, N_{c^{\prime} g}, I_{c}=I_{c^{\prime}}\right)}\right)
$$

410 where $X_{c}=\left(X_{c 1}, X_{c 2}, \ldots X_{c g}, \ldots X_{c m}\right)$ are the mutation allele read counts for cell $c$ and $N_{c}=$

$411\left(N_{c 1}, N_{c 2}, \ldots N_{c g}, \ldots N_{c m}\right)$ are the total read counts at these sites. More intuitively, if cells $c$ and $c^{\prime}$ are not

412 from the same clonal group, the numerator has larger value compared to denominator. Thus $d_{c c}^{g}$ is

413 large, indicating bigger divergence between the two cells. With further derivation (Supplementary

414 Materials), $d_{c c}^{g}$ is a function of the five following probabilities: 
$415 \quad d_{c C \prime}^{g}$

$416=f\left(P_{g} ; P\left(X_{c g} \mid N_{c g}, Z_{c g}=0\right) ; P\left(X_{c g} \mid N_{c g}, Z_{c g}=1\right) ; P\left(X_{c^{\prime} g} \mid N_{c^{\prime} g}, Z_{c^{\prime} g}=0\right) ; P\left(X_{c^{\prime} g} \mid N_{c^{\prime} g}, Z_{c^{\prime} g}=1\right)\right)$

417 where $Z_{c g} \in\{0,1\}$ is SNA indicator for cell $c$ at site $g$ and $P_{g}=P\left(Z_{g}=1\right)$ is mutation frequency across

418 the cells estimated by GATK calls.

419 In the above formula for $d_{c c l}^{g}, P\left(X_{c g} \mid N_{c g}, Z_{c g}=0\right)$ and $P\left(X_{c^{\prime} g} \mid N_{c g}, Z_{c g}=0\right)$ reflect reverse-

420 transcription/sequencing/mapping errors and rare RNA editing events, because when there is no

421 mutation (i.e. $Z_{c g}=0$ ), all mutation reads reflect such technical errors or RNA editing. Let $\epsilon$ denote the

422 combined rate of technical error and RNA editing, we have

$$
P\left(X_{c g} \mid N_{c g}, Z_{c g}=0\right) \sim \operatorname{Binomial}\left(X_{c g} \mid N_{c g}, \epsilon\right)
$$

424 For cases where there are mutations (i.e. $Z_{c g}=1$ ), the distribution of mutated read counts given total

425 read counts is modeled with a Beta Binomial distribution, which is capable of modeling technical

426 dropout and transcriptional bursting, and is supported by previous allele specific expression studies [30,

$42762]$.

$$
P\left(X_{c g} \mid N_{c g}, Z_{c g}=1\right) \sim \int_{0}^{1} \operatorname{Binomial}\left(X_{c g} \mid N_{c g}, Q_{c g}=q\right) d F(q)
$$

$$
q \sim \operatorname{Beta}\left(\alpha_{g}, \beta_{g}\right)
$$

430 where $Q_{c g}$ indicates proportion of mutated alleles expressed in cell $c$ at site $g$, with Beta distribution as

431 prior. Respectively, $\alpha_{g}$ and $\beta_{g}$ represent gene activation and deactivation rate, which are estimated

432 empirically. 
435 We cluster the cells using a kernel-based algorithm, such as hierarchical clustering. Given that there are

436 multiple sorting schemes, we leave the user to choose it. For the default-sorting scheme, we

437 recommend "ward.D" [63]. This is because $d_{c c}$, behaves like a log likelihood ratio, which should follow a

$438 \chi^{2}$ distribution when the two cells share the same subclone. The "ward.D" method has been shown to

439 work well in Euclidian space. Empirically, among different hierarchical clustering algorithms on the renal

440 cell carcinoma dataset (Figure S5) “ward.D” based hierarchical clustering performs the best.

441 To determine the number of clusters we use an intra-cluster divergence curve computed from the

442 divergence matrix. Existing software rely on AIC, BIC, or another model selection metric [64, 65].

443 However, since we only have the "distance" matrix, these traditional methods cannot be applied. Let $N_{k}$

444 be the number of cell pairs in cluster $C_{k}$ and $N$ be the total number of pairs between cells for all

445 clusters. Let $K$ be the number of clusters. The weighted sum of intra-cluster distance $W_{K}$ is

$$
W_{K}=\sum_{k=1}^{K} N_{k} \sum_{(i, j) \in C_{k}} \frac{d_{i j}}{N}
$$

447 Note that small clusters are naturally down-weighted in the above metric. DENDRO relies on visual 448 examination of the intra-cluster divergence curve $\left(W_{K}\right.$ plotted against $\left.K\right)$ to find the "elbow point", 449 which can be taken as a reasonable clustering resolution.

452 Before conducting a single cell RNA-seq experiment on a tumor sample, it is important to project how

453 subclone detection power depends on the number of cells sequenced and the coverage per cell. To 
454 facilitate experiment design, we have developed a tool, DENDROplan (Figure 2a), that predicts the

455 expected clustering accuracy by DENDRO given sequencing parameters and available bulk data for the

456 tumor. Given an assumed tree structure and a target accuracy, DENDROplan computes the necessary

457 read depth and number of cells needed.

458 As shown in Figure 2a, if bulk DNA sequencing and/or RNA sequencing data are available for the tumor

459 being studied, these data can be harnessed to make more realistic power calculations. For example, if

460 SNAs have been profiled using bulk DNA sequencing data, the set of mutations that lie in the exons of

461 annotated genes can be retrieved and used directly in constructing the spike-in data. Furthermore,

462 phylogeny construction algorithms for bulk DNA-seq data can be used to infer a putative tree structure

463 that can be used as input to DENDROplan $[5,65]$. If bulk RNA-seq data is available, the bulk expression

464 level of the mutation-carrying genes can be used to predict the expression level of the mutation in the

465 single cell data. For details, see Methods. The power analysis tool is also available at

466 https://github.com/zhouzilu/DENDRO.

Spike-in analysis

469 In our spike-in analysis, we adopt a dataset from Deng et al, which has allele specific read counts [39].

470 We further added binomial noise to mimic sequencing error. When analyzing DENDRO performance in

471 terms of various number mutation site, number of cells, proportion of cells in each clade and proportion

472 of mutations along each branch, we only take a subset of cells with similar cell types. On the other hand,

473 we utilize a mixture cell population to test the robustness of DENDRO performance with regard to

474 various expression profiles. 
477 As stated previously, DENDRO further inferred SNA after pooling the reads from all cells within each

478 cluster. Because, with our choice of thresholds, we identify SNAs in single cells with high sensitivity, the

479 "bulk" level SNAs should be a subset of the SNAs in single cells, and mutation allele counts and total

480 allele counts should provide us with enough information for SNA detection using a maximum likelihood

481 framework [66], which accounts for both sequencing error and rare RNA-editing events. Suppose $g$ is

482 the genotype (number of reference allele) at a site and assume $m$, the ploidy, equals to 2 . Then the

483 likelihood is:

$$
\mathcal{L}(g)=\frac{1}{m^{k}} \prod_{j=1}^{l}[(m-g) \epsilon+g(1-\epsilon)] \prod_{j=l+1}^{k}[(m-g)(1-\epsilon)+g \epsilon]
$$

$$
g^{*}=\underset{g}{\operatorname{argmax}}-\mathcal{L}(g)
$$

where $k$ is number of reads at a site and the first $l$ bases $(l \leq k)$ be the same to reference and the rests

487 are same to alternative allele. $\epsilon$ is the sequencing error and rare RNA-editing combined rate. Given

488 mutation profiles, DENDRO then constructs a phylogenetic tree with the neighbor-joining method,

489 which can more accurately capture the evolutionary relationship between different subclones [67] than

490 the initial tree given by hierarchical clustering.

493 We use Seurat and scDD to identify differentially expressed genes between tumors and between tumor 494 subclones [68-70]. For each comparison, we apply two different methods: MAST implemented by Seurat 495 and scDD. Genes with adjusted $p$-value $<0.05$ count as significant differentially expressed gene for each 
496 method. We further intersect these two sets of differentially expressed genes to increase robustness.

497 Subclonal mutations are annotated by ANNOVAR with default parameters and variants associated with

498 intergenic regions were discarded for downstream analysis [71]. For GO analysis, we apply Gene Set

499 Enrichment Analysis tool [52]. Hallmark gene sets serve as fundamental database with FDR q-value <

$500 \quad 0.05$ as significant.

501

502

Single cell RNA-seq of Tumor Model Derived from B16.

503 Six C57bl/6 mice were injected on both flanks with either B16 or R499: four with B16 and two with R499.

504 Two of the mice implanted with B16 were treated with 200 ug of anti-PD1 per mouse on Days 5, 8 and

505 11. On Day 15, all tumors were harvested and made into single cell suspension. 100,000 CD45 negative

506 tumor cells were sorted on Aria to enrich for live tumor cells and loaded on SMARTer ICELL8 cx Single-

507 Cell System prior to full length single cell RNA-sequencing library preparation using Smart-seq following

508 manufacturer's recommendations. 460 cells and 11531 genes passed standard QC and were retained for

509 downstream analysis.

510

511 Neoantigen prediction.

512 Based on gene expression from RNA-seq data, variants from unexpressed transcripts are removed. The

513 MHC-I binding affinities of variants are then predicted using NetMHC version 4.0 for $\mathrm{H}-2-\mathrm{Kb}$ and $\mathrm{H}-2-\mathrm{Db}$

514 using peptide lengths from 8 to 11 [72]. Given subclonal mutation profile, we further assign the

515 neoantigens to each subclone. 


\section{List of abbreviations}

518 SNA: Single nucleotide alteration

519 scDNA-seq: Single-cell DNA sequencing

520 scRNA-seq: Single-cell RNA sequencing

521 PDX: Patient-derived xenograft

522 TPM: Transcripts per kilobase million

523 ICB: Immune checkpoint blockade

524 TMB: Tumor mutational burden

525

\section{Declarations}

\section{Acknowledgements}

528 We thank Dr. Nan Lin for informing useful tools for biological insight analysis, and Dr. Kai Tan and Dr.

529 Mingyao Li for helpful comments and suggestions.

\section{$530 \quad$ Funding}

531 This work was supported by National Institutes of Health (NIH) grant 1P01CA210944-01 to AM and BX,

532 5R01-HG006137-07 to ZZ and to NRZ, and 1U2CCA233285-01 to NRZ. 
534 DENDRO is an open-source R package available at https://github.com/zhouzilu/DENDRO with license

535 GPL-3.0.

Authors' contributions

537 ZZ and NRZ formulated the model. ZZ developed and implemented the algorithm and conducted all

538 computational analyses. BX and AM designed the immunotherapy case study. BX performed the

539 experiments of the immunotherapy case. ZZ, BX and NRZ wrote the manuscript. All authors read and

540 approved the final manuscript.

\section{Competing interests}

542 The authors declare that they have no competing interests.

544 Not applicable.

$546 \quad$ Not applicable.

547 1. Gamazon, E.R. and B.E. Stranger, The impact of human copy number variation on gene mutations in BUB1B. Nat Genet, 2004. 36(11): p. 1159-61.

3. Vicente-Duenas, C., et al., Epigenetic Priming in Cancer Initiation. Trends Cancer, 2018. 4(6): p. 408-417.

4. Burrell, R.A., et al., The causes and consequences of genetic heterogeneity in cancer evolution. Nature, 2013. 501(7467): p. 338-45. 
5. Jiang, Y., et al., Assessing intratumor heterogeneity and tracking longitudinal and spatial clonal evolutionary history by next-generation sequencing. Proc Natl Acad Sci U S A, 2016. 113(37): p. E5528-37.

6. Deshwar, A.G., et al., PhyloWGS: reconstructing subclonal composition and evolution from whole-genome sequencing of tumors. Genome Biol, 2015. 16: p. 35.

7. Zare, $\mathrm{H}$., et al., Inferring clonal composition from multiple sections of a breast cancer. PLoS Comput Biol, 2014. 10(7): p. e1003703.

8. Carter, S.L., et al., Absolute quantification of somatic DNA alterations in human cancer. Nat Biotechnol, 2012. 30(5): p. 413-21.

9. $\quad \mathrm{Li}, \mathrm{B}$. and J.Z. Li, A general framework for analyzing tumor subclonality using SNP array and DNA sequencing data. Genome Biol, 2014. 15(9): p. 473.

10. Oesper, L., A. Mahmoody, and B.J. Raphael, THetA: inferring intra-tumor heterogeneity from high-throughput DNA sequencing data. Genome Biol, 2013. 14(7): p. R80.

11. Ha, G., et al., TITAN: inference of copy number architectures in clonal cell populations from tumor whole-genome sequence data. Genome Res, 2014. 24(11): p. 1881-93.

12. Miller, C.A., et al., SciClone: inferring clonal architecture and tracking the spatial and temporal patterns of tumor evolution. PLoS Comput Biol, 2014. 10(8): p. e1003665.

13. Navin, N.E., The first five years of single-cell cancer genomics and beyond. Genome Res, 2015. 25(10): p. 1499-507.

14. Navin, N., et al., Tumour evolution inferred by single-cell sequencing. Nature, 2011. 472(7341): p. 90-4.

15. Wang, Y., et al., Clonal evolution in breast cancer revealed by single nucleus genome sequencing. Nature, 2014. 512(7513): p. 155-60.

16. Gao, R., et al., Punctuated copy number evolution and clonal stasis in triple-negative breast cancer. Nat Genet, 2016. 48(10): p. 1119-30.

17. Picelli, S., et al., Smart-seq2 for sensitive full-length transcriptome profiling in single cells. Nat Methods, 2013. 10(11): p. 1096-8.

18. Klein, A.M., et al., Droplet barcoding for single-cell transcriptomics applied to embryonic stem cells. Cell, 2015. 161(5): p. 1187-1201.

19. Patel, A.P., et al., Single-cell RNA-seq highlights intratumoral heterogeneity in primary glioblastoma. Science, 2014. 344(6190): p. 1396-401.

20. Chung, W., et al., Single-cell RNA-seq enables comprehensive tumour and immune cell profiling in primary breast cancer. Nat Commun, 2017. 8: p. 15081.

21. Kim, K.T., et al., Application of single-cell RNA sequencing in optimizing a combinatorial therapeutic strategy in metastatic renal cell carcinoma. Genome Biol, 2016. 17: p. 80.

22. Tirosh, I., et al., Dissecting the multicellular ecosystem of metastatic melanoma by single-cell RNA-seq. Science, 2016. 352(6282): p. 189-96.

23. Jerby-Arnon, L., et al., A Cancer Cell Program Promotes T Cell Exclusion and Resistance to Checkpoint Blockade. Cell, 2018. 175(4): p. 984-997 e24.

24. Tirosh, I., et al., Single-cell RNA-seq supports a developmental hierarchy in human oligodendroglioma. Nature, 2016. 539(7628): p. 309-313.

25. Venteicher, A.S., et al., Decoupling genetics, lineages, and microenvironment in IDHmutant gliomas by single-cell RNA-seq. Science, 2017. 355(6332). 
26. $\mathrm{Li}, \mathrm{H}$., et al., Reference component analysis of single-cell transcriptomes elucidates cellular heterogeneity in human colorectal tumors. Nat Genet, 2017. 49(5): p. 708-718.

27. van Galen, P., et al., Single-Cell RNA-Seq Reveals AML Hierarchies Relevant to Disease Progression and Immunity. Cell, 2019. 176(6): p. 1265-1281 e24.

28. Nam, A.S., et al., Somatic mutations and cell identity linked by Genotyping of Transcriptomes. Nature, 2019. 571(7765): p. 355-360.

29. Raj, A., et al., Stochastic mRNA synthesis in mammalian cells. PLoS Biol, 2006. 4(10): p. e309.

30. Jiang, Y., N.R. Zhang, and M. Li, SCALE: modeling allele-specific gene expression by singlecell RNA sequencing. Genome Biol, 2017. 18(1): p. 74.

31. Padovan-Merhar, O., et al., Single mammalian cells compensate for differences in cellular volume and DNA copy number through independent global transcriptional mechanisms. Mol Cell, 2015. 58(2): p. 339-52.

32. Zafar, $\mathrm{H}$., et al., Monovar: single-nucleotide variant detection in single cells. Nat Methods, 2016. 13(6): p. 505-7.

33. Piskol, R., G. Ramaswami, and J.B. Li, Reliable identification of genomic variants from RNA-seq data. Am J Hum Genet, 2013. 93(4): p. 641-51.

34. Brennecke, P., et al., Accounting for technical noise in single-cell RNA-seq experiments. Nat Methods, 2013. 10(11): p. 1093-5.

35. Pierson, E. and C. Yau, ZIFA: Dimensionality reduction for zero-inflated single-cell gene expression analysis. Genome Biol, 2015. 16: p. 241.

36. Vallejos, C.A., J.C. Marioni, and S. Richardson, BASiCS: Bayesian Analysis of Single-Cell Sequencing Data. PLoS Comput Biol, 2015. 11(6): p. e1004333.

37. Ding, B., et al., Normalization and noise reduction for single cell RNA-seq experiments. Bioinformatics, 2015. 31(13): p. 2225-7.

38. Qiu, X., et al., Single-cell mRNA quantification and differential analysis with Census. Nat Methods, 2017. 14(3): p. 309-315.

39. Deng, Q., et al., Single-cell RNA-seq reveals dynamic, random monoallelic gene expression in mammalian cells. Science, 2014. 343(6167): p. 193-6.

40. Eirew, P., et al., Dynamics of genomic clones in breast cancer patient xenografts at single-cell resolution. Nature, 2015. 518(7539): p. 422-6.

41. Gerlinger, M., et al., Intratumor heterogeneity and branched evolution revealed by multiregion sequencing. N Engl J Med, 2012. 366(10): p. 883-892.

42. Shi, Y.J., et al., Intratumoral Heterogeneity in Breast Cancer: A Comparison of Primary and Metastatic Breast Cancers. Oncologist, 2017. 22(4): p. 487-490.

43. Ribas, A. and J.D. Wolchok, Cancer immunotherapy using checkpoint blockade. Science, 2018. 359(6382): p. 1350-1355.

44. Schumacher, T.N. and R.D. Schreiber, Neoantigens in cancer immunotherapy. Science, 2015. 348(6230): p. 69-74.

45. Rizvi, N.A., et al., Cancer immunology. Mutational landscape determines sensitivity to PD-1 blockade in non-small cell lung cancer. Science, 2015. 348(6230): p. 124-8.

46. Tumeh, P.C., et al., PD-1 blockade induces responses by inhibiting adaptive immune resistance. Nature, 2014. 515(7528): p. 568-71. 
641 47. Twyman-Saint Victor, C., et al., Radiation and dual checkpoint blockade activate non-

642

643

644

645

646

647

648

649

650

651

652

653

654

655

656

657

658

659

660

661

662

663

664

665

666

667

668

669

670

671

672

673

674

675

676

677

678

679

680

681

682

683 redundant immune mechanisms in cancer. Nature, 2015. 520(7547): p. 373-7.

48. Benci, J.L., et al., Opposing Functions of Interferon Coordinate Adaptive and Innate Immune Responses to Cancer Immune Checkpoint Blockade. Cell, 2019. 178(4): p. 933948 e14.

49. Patel, S.A. and A.J. Minn, Combination Cancer Therapy with Immune Checkpoint Blockade: Mechanisms and Strategies. Immunity, 2018. 48(3): p. 417-433.

50. Goodman, A.M., et al., Tumor Mutational Burden as an Independent Predictor of Response to Immunotherapy in Diverse Cancers. Mol Cancer Ther, 2017. 16(11): p. 25982608.

51. Rosenthal, R., et al., Neoantigen-directed immune escape in lung cancer evolution. Nature, 2019. 567(7749): p. 479-485.

52. Subramanian, A., et al., Gene set enrichment analysis: a knowledge-based approach for interpreting genome-wide expression profiles. Proc Natl Acad Sci U S A, 2005. 102(43): p. 15545-50.

53. Parker, J.S., et al., Supervised risk predictor of breast cancer based on intrinsic subtypes. J Clin Oncol, 2009. 27(8): p. 1160-7.

54. Zhang, J.Y., et al., Critical protein GAPDH and its regulatory mechanisms in cancer cells. Cancer Biol Med, 2015. 12(1): p. 10-22.

55. Tarrado-Castellarnau, M., et al., Glyceraldehyde-3-phosphate dehydrogenase is overexpressed in colorectal cancer onset. Translational Medicine Communications, 2017. 2(1): p. 6.

56. Mann, M., V. Cortez, and R.K. Vadlamudi, Epigenetics of estrogen receptor signaling: role in hormonal cancer progression and therapy. Cancers (Basel), 2011. 3(3): p. 1691-707.

57. Green, K.A. and J.S. Carroll, Oestrogen-receptor-mediated transcription and the influence of co-factors and chromatin state. Nat Rev Cancer, 2007. 7(9): p. 713-22.

58. Dreijerink, K.M., et al., Menin links estrogen receptor activation to histone H3K4 trimethylation. Cancer Res, 2006. 66(9): p. 4929-35.

59. Kim, H., et al., Requirement of histone methyltransferase SMYD3 for estrogen receptormediated transcription. J Biol Chem, 2009. 284(30): p. 19867-77.

60. Dobin, A., et al., STAR: ultrafast universal RNA-seq aligner. Bioinformatics, 2013. 29(1): p. $15-21$.

61. McKenna, A., et al., The Genome Analysis Toolkit: a MapReduce framework for analyzing next-generation DNA sequencing data. Genome Res, 2010. 20(9): p. 1297-303.

62. Skelly, D.A., et al., A powerful and flexible statistical framework for testing hypotheses of allele-specific gene expression from RNA-seq data. Genome Res, 2011. 21(10): p. 172837.

63. Ward, J.H., Hierarchical Grouping to Optimize an Objective Function. Journal of the American Statistical Association, 1963. 58(301): p. 236-\&.

64. Goutte, C., et al., Feature-space clustering for fMRI meta-analysis. Hum Brain Mapp, 2001. 13(3): p. 165-83.

65. Urrutia, E., et al., Integrative pipeline for profiling DNA copy number and inferring tumor phylogeny. Bioinformatics, 2018. 34(12): p. 2126-2128. 
684 66. Li, B., et al., A likelihood-based framework for variant calling and de novo mutation

685 detection in families. PLoS Genet, 2012. 8(10): p. e1002944.

686 67. Schliep, K.P., phangorn: phylogenetic analysis in R. Bioinformatics, 2011. 27(4): p. 592-3.

687 68. Finak, G., et al., MAST: a flexible statistical framework for assessing transcriptional changes and characterizing heterogeneity in single-cell RNA sequencing data. Genome Biol, 2015. 16: p. 278.

690 69. Korthauer, K.D., et al., A statistical approach for identifying differential distributions in single-cell RNA-seq experiments. Genome Biol, 2016. 17(1): p. 222.

693 technologies, and species. Nat Biotechnol, 2018. 36(5): p. 411-420.

71. Wang, K., M. Li, and H. Hakonarson, ANNOVAR: functional annotation of genetic variants from high-throughput sequencing data. Nucleic Acids Res, 2010. 38(16): p. e164.

72. Karosiene, E., et al., NetMHCcons: a consensus method for the major histocompatibility complex class I predictions. Immunogenetics, 2012. 64(3): p. 177-86. 
bioRxiv preprint doi: https://doi.org/10.1101/457622; this version posted October 17, 2019. The copyright holder for this preprint (which was not A certified by peer review) is the author/funder, wh\$h has granted bioRxiv a license to display the preprint in perpetuity It is made available under
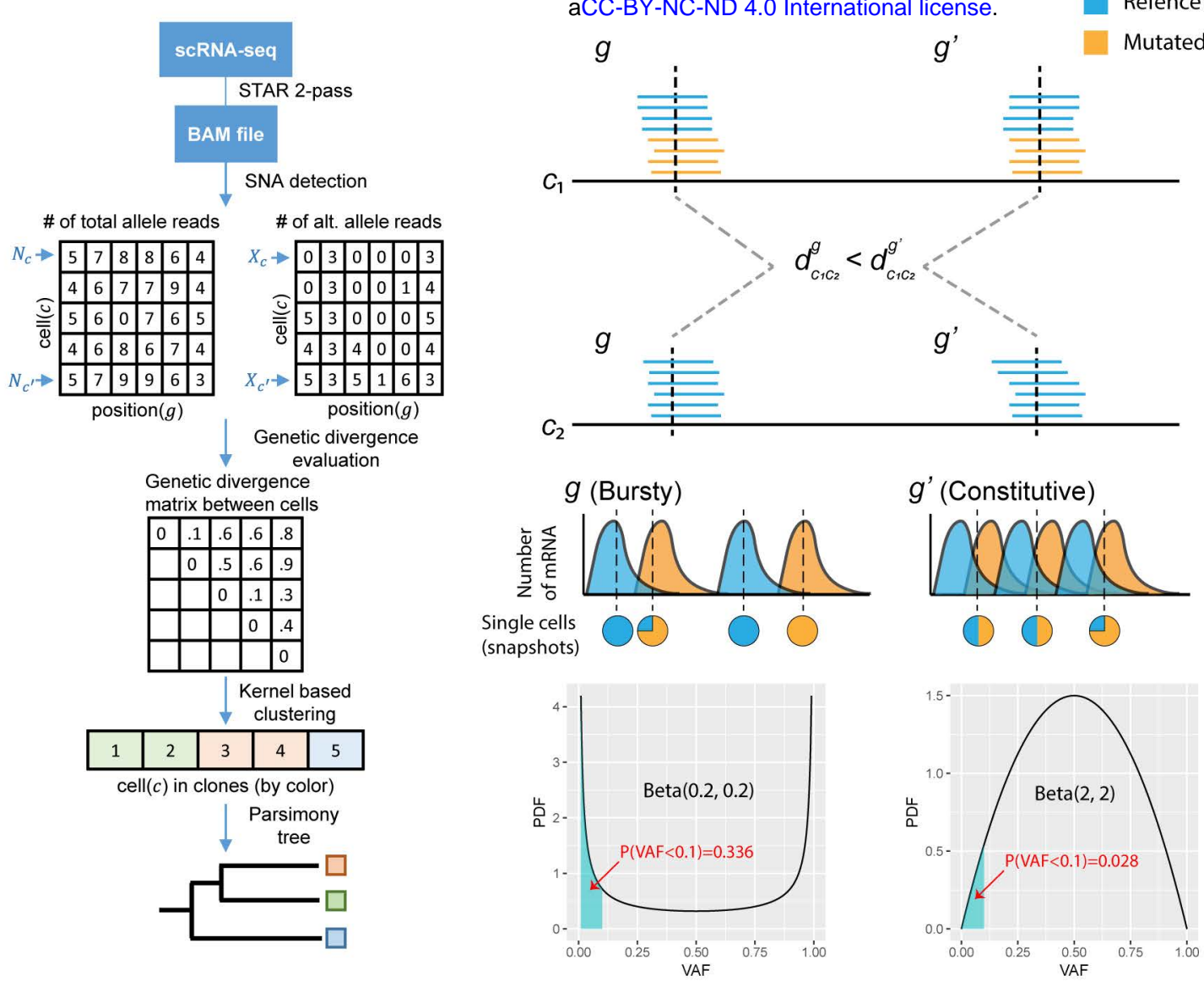

Figure 1. DENDRO analysis pipeline and genetic divergence evaluation. a DENDRO analysis pipeline overview. b Statistical model for genetic divergence evaluation function. In this example, we show relationship of cell $c_{1}$ and $c_{2}$ with gene $g$ and $g$ ' in terms of their genetic divergence. Middle panels shows the stochasticity of cell expression and bottom panels illustrates the distribution of variants allele frequency across cells for these two genes, indicating that gene $g$ is a bursty gene and $g^{\prime}$ is a constitutive gene. 
A

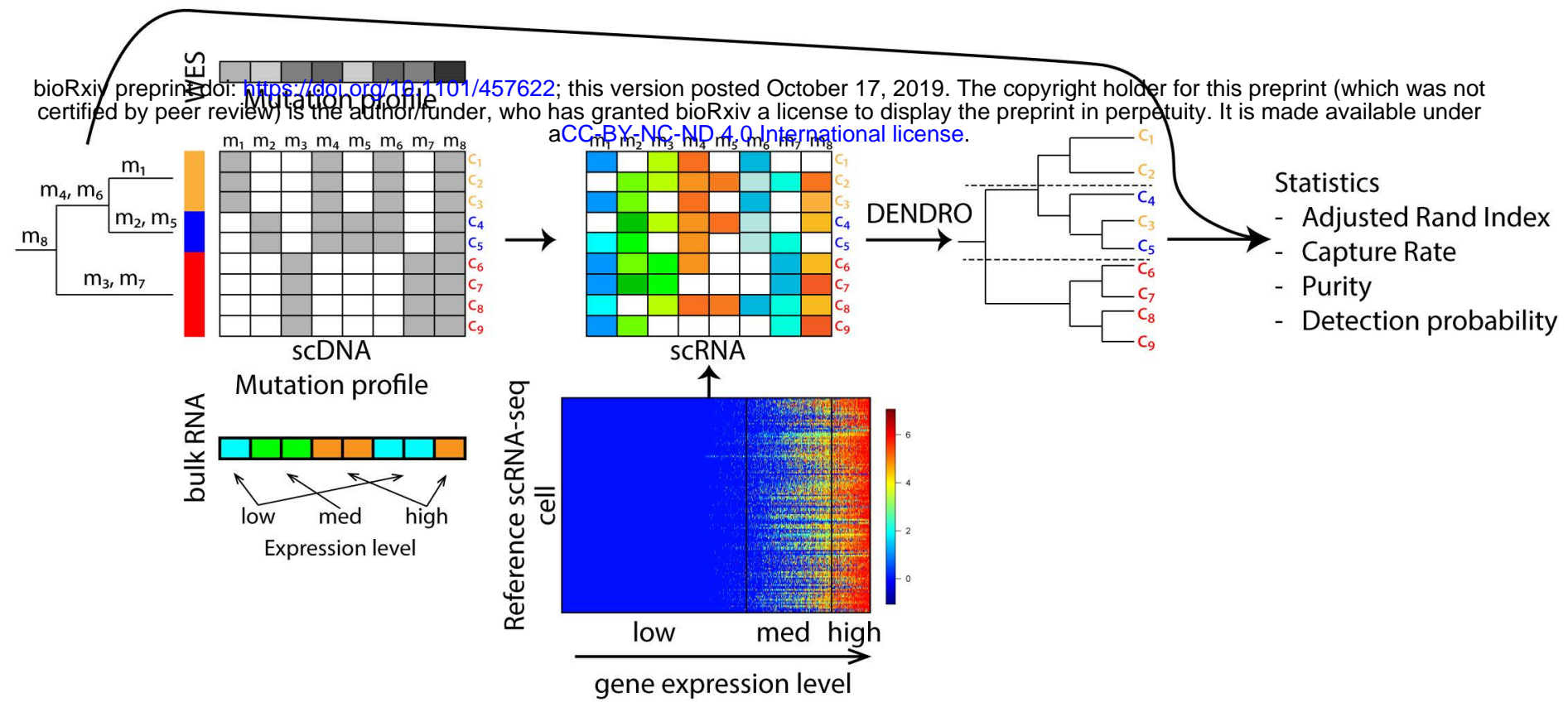

B
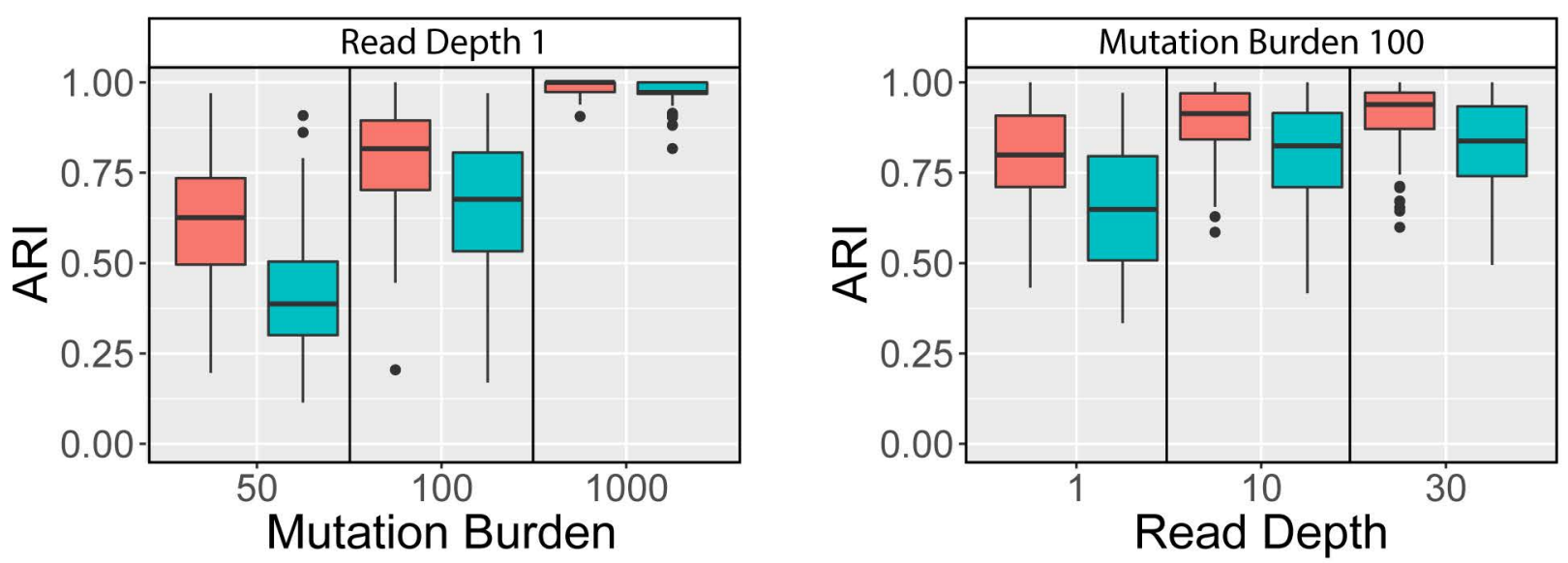

Simulation Tree

C

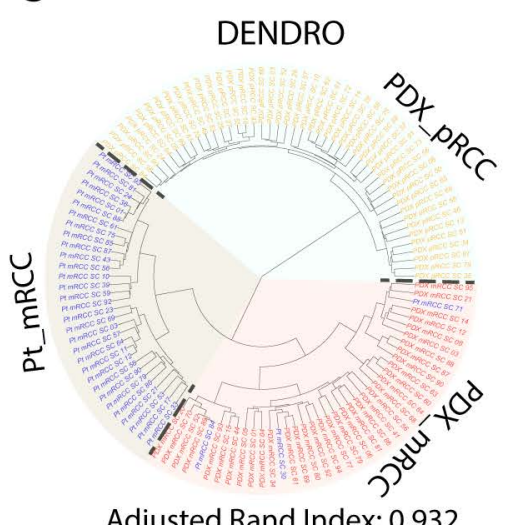

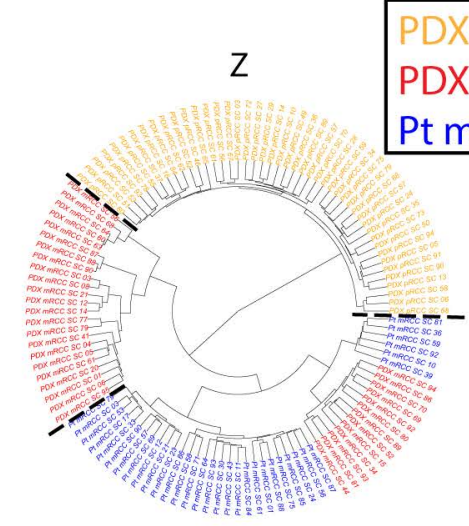

Adjusted Rand Index: 0.754

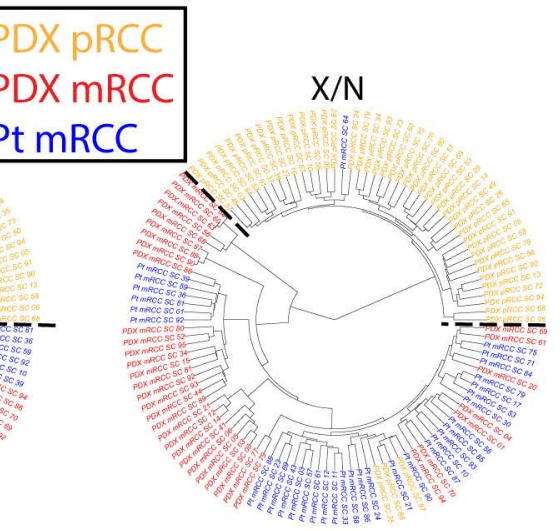

Adjusted Rand Index: 0.519

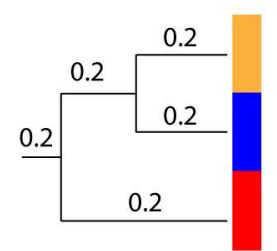

DENDRO

$X / N$

Figure 2. DENDRO accuracy assessment. a The overall spike-in analysis pipeline. Three statistics measure the accuracy of DENDRO - adjusted Rand index (global accuracy, 1 indicating perfect classification), capture rate (subclone specific accuracy, out of all cells from the clade, how many of them are correctly assigned) and purity (subclone specific accuracy, out of all cells from the detected cluster, how many are actually from the clade). b Cluster accuracy via spike-in studies.Various parameters show effects on cluster accuracy (measured by adjusted Rand index) based on tree structure on the right. Left panel: effect of mutation read depth. Right panel: effect of total mutation burden. $\mathbf{c}$ Evaluation of DENDRO on a renal cell carcinoma and its metastasis. (Left to right) (1) DENDRO clustering result from primary and metastatic renal cell carcinoma dataset. Background colors represent DEDRO clustering result. (2) Clustering of the same dataset using $Z$ matrix (indicator matrix, $z_{i j}=1$ when detected a mutation for cell $i$ at site $j$ by GATK tool). (3) Clustering of the same dataset using $X / N$ matrix (mutation allele frequency matrix) (4) Clustering of the same dataset using expression $(\log \mathrm{T}$ PM). 


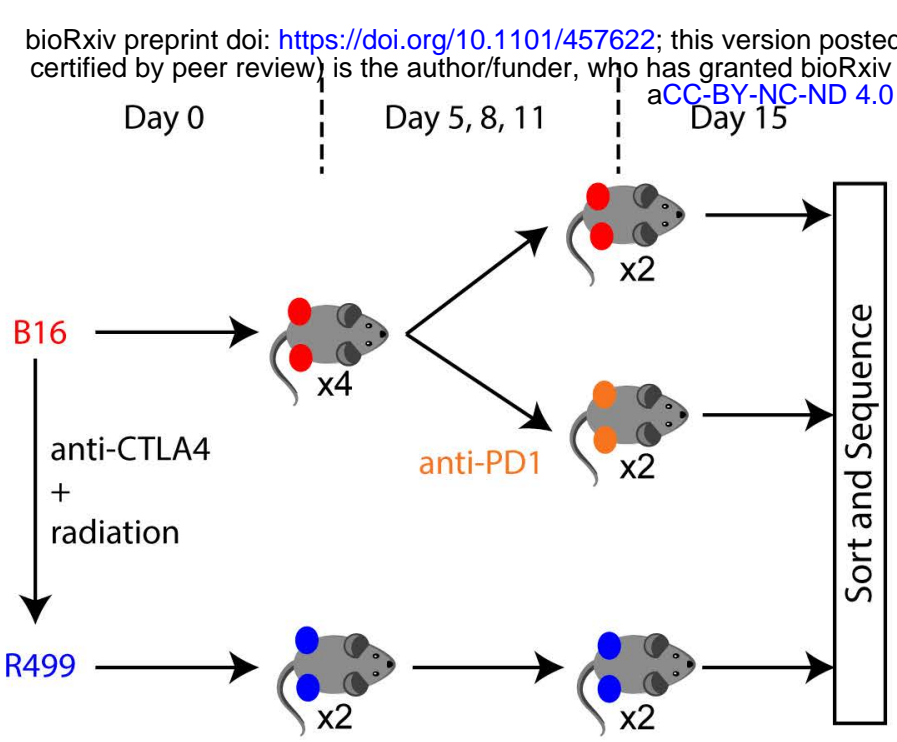

C

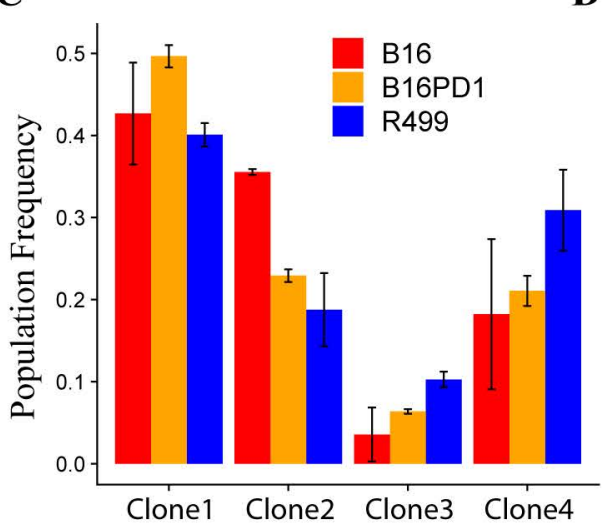

D

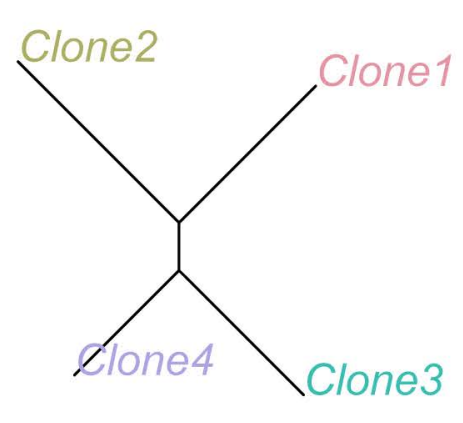

$\mathbf{E}$

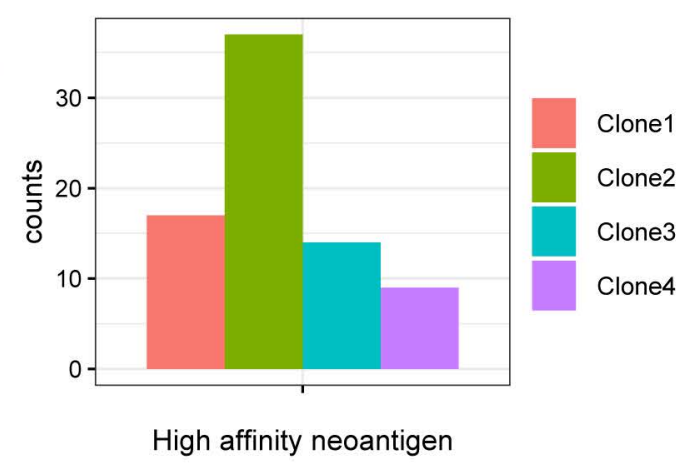

Figure 3. Clonal composition alternations with anti-PD1 treatments and cell lines. a Experimental overview. For each condition at Day 15, we have two biological replicates. There are total 600 cells from 6 tumors sequenced. b DENDRO cluster result. No clone is exclusively associated with any tumor condition. $\mathbf{c}$ Frequencies of the subclonal populations in B16, B16PD1 and R499. d Neighbor joining phylogenetic tree given detected subclones. e Number of high affinity neoantigen predicted for each clone. Clone 2 have the highest number of neoantigen. 
bioRxiv preprint doi: https://doi.org/10.1101/457622; this version posted October 17, 2019. The copyright holder for this preprint (which was not certified by peer review) is the auB Oeffender, who has granted bioRxiv a license to display the preprint in perpetuity. It is made available under

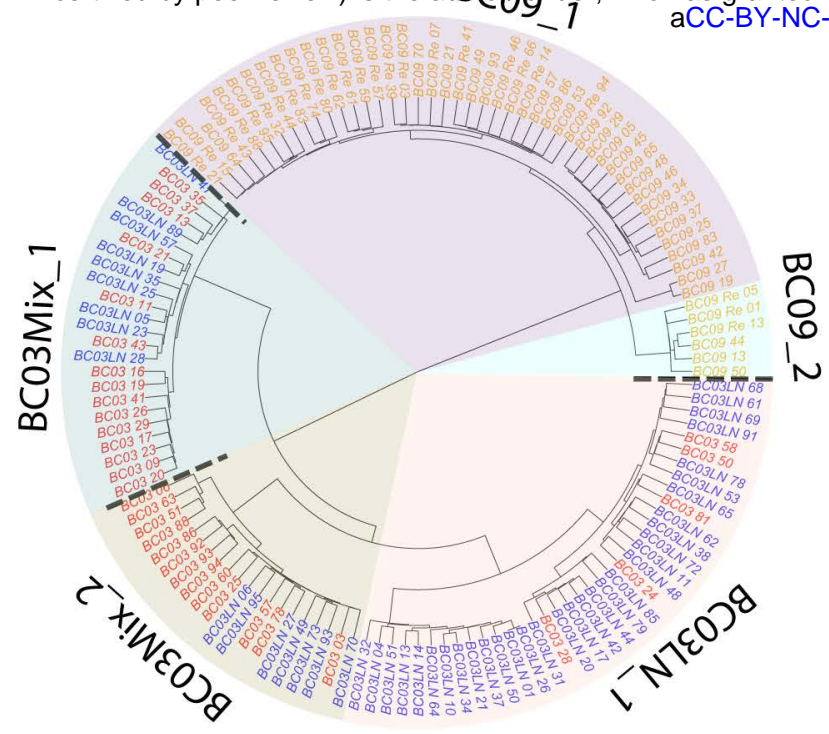

Adjusted Rand Index: 1.000

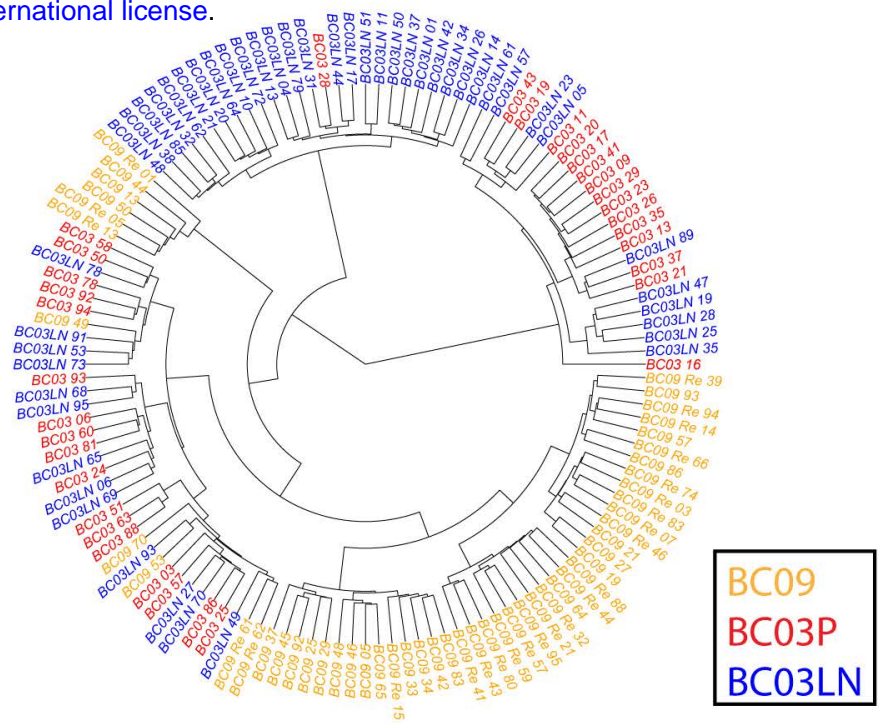

Adjusted Rand Index: -0.0014
C

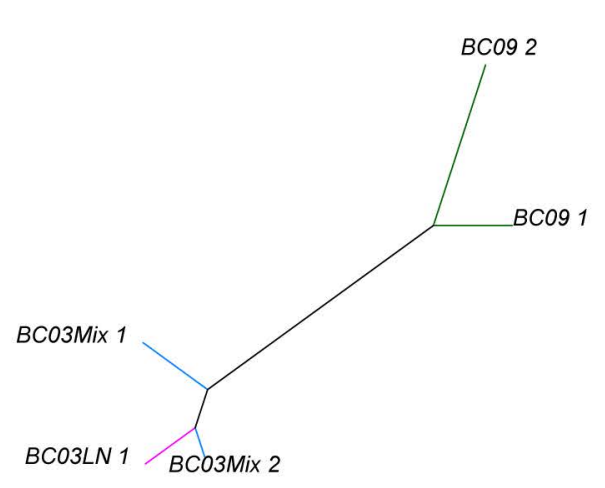

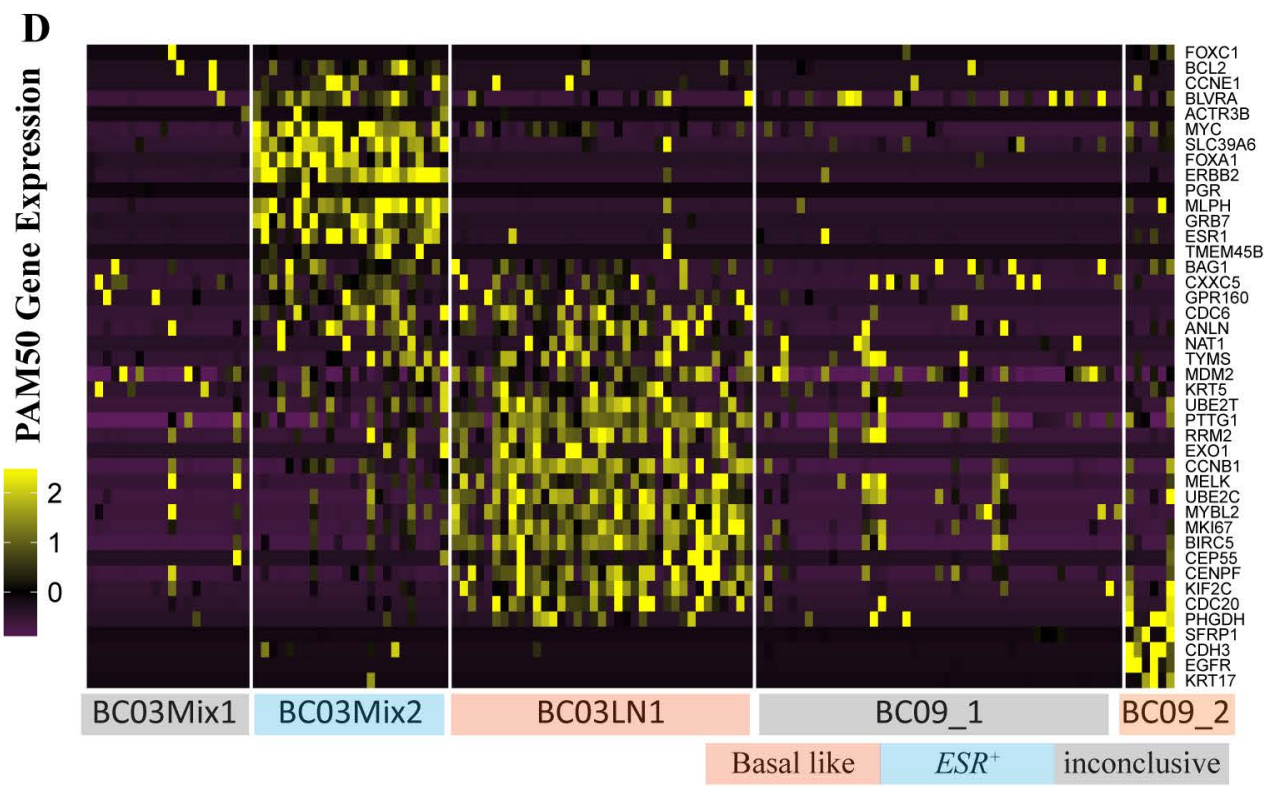

Figure 4. Analysis of scRNA-seq dataset of primary breast cancer. a DENDRO cluster result for primary breast cancer dataset (Chung et al., 2017). b Hierarchical clustering result for the same dataset based on expression $(\log$ TPM). (dashlines indicate cluster boundaries). c Neighbor joining phylogenetic tree given detected subclones for breast cancer dataset. d PAM50 gene panel expression shows breast cancer subtypes of each subclone. 